\section{Clinical features and electrocardiogram parameters in Parkinson's disease}

\author{
Hitoshi Mochizuki, Nobuyuki Ishii, \\ Kazutaka Shiomi, Masamitsu Nakazato \\ Division of Neurology, Respirology, \\ Endocrinology and Metabolism, \\ Department of Internal Medicine, \\ University of Miyazaki, Japan
}

\begin{abstract}
We investigated the relation between clinical features and electrocardiogram (ECG) parameters in patients with Parkinson's disease (PD). One hundred and fifty-six PD patients were enrolled. Their clinical features [body mass index (BMI), age, disease duration, and disease stage] and ECG parameters [RR, PR, QRS, and QT intervals, and heart rate-corrected QT (QTc)] were analyzed. BMI was positively correlated with the PR and QRS intervals in patients with PD. The QRS interval was positively correlated with disease duration and Hoehn and Yahr stage, and the QT interval and QTc were positively correlated with age. Clinical features and ECG parameters are likely to be closely associated with each other. Several ECG parameters reflect autonomic dysfunction or disease progression. Clinicians should pay more attention to ECG parameters in the treatment of PD patients.
\end{abstract}

\section{Introduction}

Several previous papers indicated reduced uptake in cardiac 123Imetaiodobenzylguanidine scintigraphy (MIBG) in patients with Parkinson's disease (PD). ${ }^{1}$ Recently, prolongation of the PR interval on electrocardiogram (ECG) was reported to reflect the abnormal MIBG findings in PD. ${ }^{2}$ In addition, an increased body mass index (BMI) was associated with autonomic dysfunction in PD as determined using the MIBG method. ${ }^{3}$ In this study, we investigated the relation between clinical features, including BMI, and ECG parameters in patients with PD.

\section{Materials and Methods}

This was an observational, cross-sectional study. From April 2011 to December 2016, 160 consecutive possible PD patients who were naïve to anti-parkinsonian drugs were admitted to our hospital. Their diagnoses were done according to the National Institute of Neurological Disorders and Stroke diagnostic criteria for PD. 4 After ECG recording, it was confirmed that all patients exhibited a beneficial response to L-DOPA. Their clinical features (BMI, age, disease duration, and disease stage) and ECG parameters were analyzed. Disease stage was defined based on the Hoehn and Yahr (H-Y) scale. ${ }^{5}$ Four patients were excluded from the study because of diabetes mellitus. ${ }^{6}$ No subjects had heart disease or received antidepressants, antiemetics, or atypical antipsychotics, all of which are factors that could affect ECG parameters. The final study population consisted of 156 patients with PD (female 90, male 66). A standard 12-lead ECG was recorded with an ECG machine (FCP-7541; Fukuda Denshi, Tokyo, Japan) in all subjects between $2 \mathrm{pm}$ and $3 \mathrm{pm}$. The RR, PR, QRS, and QT intervals were measured, and QTc was calculated by $\mathrm{QT} /(\mathrm{RR})^{1 / 2}$. Written informed consent was obtained from all the participants. This study protocol was approved by the local ethics committee and was carried out in accordance to the Declaration of Helsinki.

The correlations between clinical features and ECG parameters were analyzed using Spearman's rank correlation coefficient. The significance level was set at $\mathrm{P}=0.05$. SPSS version 22 software was used for statistical analysis.

\section{Results}

The BMI, age (years), disease duration (months), and H-Y stage of the 156 PD patients, expressed as mean $\pm \mathrm{SD}$, were $22.8 \pm 3.2,68.5 \pm 8.7,22.4 \pm 17.5$, and $2.2 \pm 0.8$, respectively. Table 1 shows the relations between clinical features and ECG parameters. BMI was positively correlated with the PR and QRS intervals in patients with PD (Figure 1A and B), regardless of disease duration and severity. The QRS interval was positively correlated with disease duration and H-Y stage (Figure 1C), and the QT interval and QTc were positively correlated with age (Figure 1D).

\section{Discussion}

This study demonstrated three main findings. First, BMI was positively correlated with the PR and QRS intervals. Second, the QRS interval was positively correlated with disease duration and H-Y stage. Third, QTc was positively correlated with age.
Correspondence: Hitoshi Mochizuki, Division of Neurology, Respirology, Endocrinology and Metabolism, Department of Internal Medicine, University of Miyazaki, 5200 Kihara, Kiyotake, Miyazaki, 889-1692, Japan. Tel: +81-985-85-2965 - Fax: +81-985-85-1869. E-mail: mochizuki-h@umin.net

Key words: Parkinson's disease; Electrocardiography; Atrioventricular conduction.

Conflict of Interest: The authors declare no potential conflict of interest.

Ethical approval: All procedures performed in studies involving human participants were in accordance with the ethical standards of the institution and with the 1964 Helsinki declaration and its later amendments.

Informed consent: Informed consent was obtained from all individual participants included in the study.

Contributions: HM, design and coordination of the study, analysis and interpretation of the data, collection of the material, drafting of the manuscript; NI, analysis and interpretation of the data, collection of the material, drafting of the manuscript; $\mathrm{KS}$ and $\mathrm{MN}$, interpretation of the data, drafting of the manuscript.

Received for publication: 15 August 2017. Accepted for publication: 11 October 2017.

This work is licensed under a Creative Commons Attribution NonCommercial 4.0 License (CC BY-NC 4.0).

(C) Copyright H. Mochizuki et al., 2017 Licensee PAGEPress, Italy

Neurology International 2017; 9:7356 doi:10.4081/ni.2017.7356

In our previous studies of patients with $\mathrm{PD}$, the PR interval was correlated with autonomic dysfunction, and autonomic dysfunction was associated with BMI increase. ${ }^{2,3}$ The results of the current study indicated a positive correlation between BMI and the PR and QRS intervals. In a large-scale study of normal subjects, prolongation of both the PR and QRS intervals were reported in obese people. ${ }^{7}$ Our results suggest a similar relation in PD patients, and indicate that autonomic dysfunction, BMI increase, and prolonged PR and QRS intervals are likely to be closely associated with each other in PD.

The QRS interval was positively correlated with disease duration and $\mathrm{H}-\mathrm{Y}$ stage in the current study. This interval represents the near-simultaneous activation of the right and left ventricles. A pathological study of 
Table 1. Correlations between clinical features and electrocardiogram parameters.

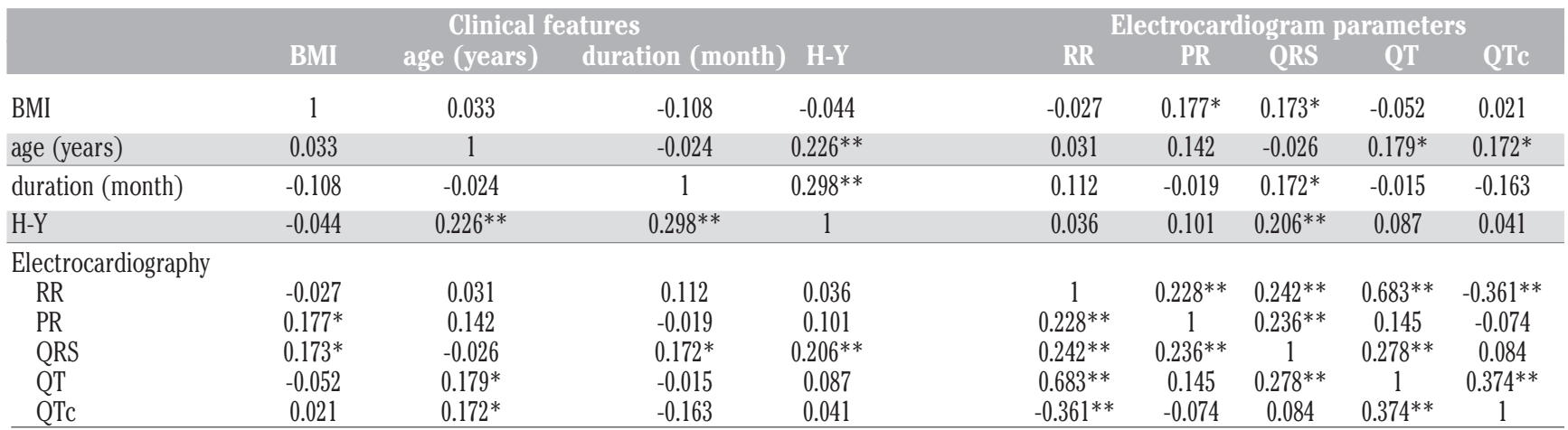

The results indicate Spearman's rank correlation coefficients (rho). ${ }^{*}, \mathrm{P}<0.05 ;{ }^{* *}, \mathrm{P}<0.01$. BMI, body mass index; H-Y, Hoehn and Yahr scale.

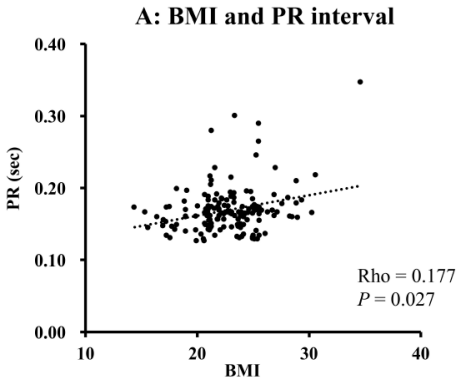

C: H-Y scale and QRS interval

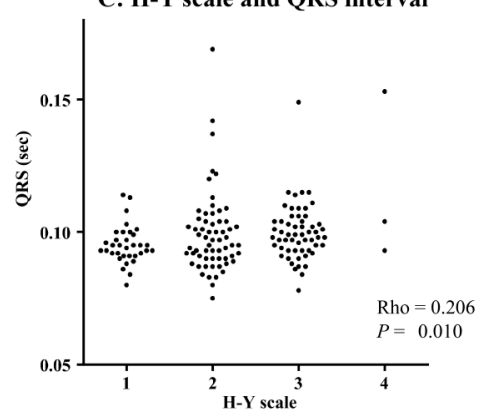

B: BMI and QRS interval

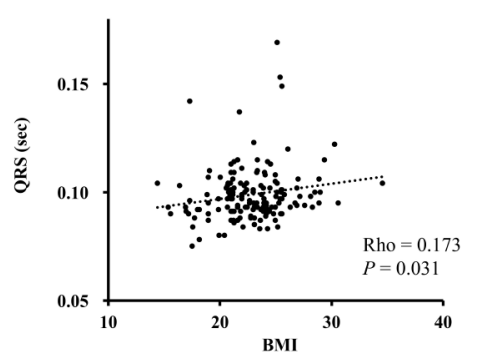

D: Age and QTe

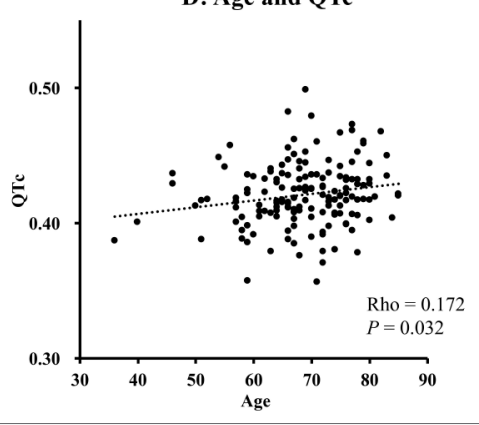

Figure 1. Scatter diagrams showing the relationship between clinical features and electrocardiogram parameters. Body mass index was positively correlated with PR (A) and QRS (B) intervals. QRS interval was positively correlated with Hoehn and Yahr (C) stage, and QTc was positively correlated with age (D).

PD found that cardiac sympathetic dysfunction affected the heart's conduction system, including the His bundle, the principal Tawara bundles, and the Purkinje fibers. ${ }^{8}$ Sympathetic nerve dysfunction may cause a prolonged QRS interval in PD as the disease progresses. ECG analysis in a largescale study of normal subjects revealed that QTc interval increased with age, but the statistical analysis was not performed. ${ }^{9}$ Our results indicated that QTc was positively correlated with age in PD patients. In another study, QTc was more prolonged in PD patients than in normal subjects. 10 Taken together, these findings suggest that elderly PD patients may exhibit particularly prolonged QTc interval. Over the past decade, considerable attention has been paid to sudden death in PD. In a large-scale cohort study, mortality in 10 out of $131 \mathrm{PD}$ patients $(7.6 \%)$ was due to sudden death. ${ }^{11}$ Prolongation of the QTc interval increases the risk of sudden cardiac death. ${ }^{12}$ To decrease this risk, attention should be paid to the QTc interval during the medical treatment of elderly PD patients.

\section{Conclusions}

Clinical features and ECG parameters are likely to be closely associated with each other. Several ECG parameters reflect autonomic dysfunction or disease progression. Clinicians should pay more attention to ECG parameters in the treatment of PD patients.

\section{References}

1. Orimo S, Ozawa E, Nakade S, et al. 123Imetaiodobenzylguanidine myocardial scintigraphy in Parkinson's disease. J Neurol Neurosurg Psych 1999;67:189-94.

2. Mochizuki H, Ebihara Y, Ugawa Y, et al. PR prolongation and cardiac 123I-MIBG uptake reduction in Parkinson's disease. Eur Neurol 2015;74:107-11.

3. Mochizuki H, Taniguchi A, Nakazato Y, et al. Increased body mass index associated with autonomic dysfunction in Parkinson's disease. Parkinson Relat Disord 2016;24:129-31.

4. Gelb DJ, Oliver E, Gilman S. Diagnostic criteria for Parkinson disease. Arch Neurol 1999;56:33-9.

5. Hoehn MM, Yahr MD. Parkinsonism: onset, progression, and mortality. Neurology 1967;17:427-42.

6. American diabetes association. Diagnosis and classification of diabetes mellitus. Diabetes Care 2013;36:67-74.

7. Frank S, Colliver JA, FrankA. The electrocardiogram in obesity: statistical analysis of 1,029 patients. J Am Coll Cardiol 1986;7:295-9.

8. Ghebremedhin E, Tredici KD, Langston JW, et al. Diminished tyrosine hydroxylase immunoreactivity in the cardiac conduction system and myocardium in Parkinson's disease: an anatomical study. Acta Neuropathol 2009;118:777-84.

9. Rijnbeek PR, van Herpen G, Bots ML, et al. Normal values of the electrocardiogram for ages 16-90 years. J Electrocard 2014;47:91421.

10. Oka H, Mochio S, Sato H, et al. Prolongation of QTc interval in patients with Parkinson's disease. Eur Neurol 1997;37:186-9.

11. Sato K, Hatano T, Yamashiro K, et al. Prognosis of Parkinson's disease: time to stage III, IV, V, and to motor fluctuations. Mov Disord 2006;21:1384-95.

12. Straus SM, Kors JA, De Bruin ML, et al. Prolonged QTc interval and risk of sudden cardiac death in a population of older adults. J Am Coll Cardiol 2006;47:362-7. 